\title{
In vitro assessment of stearyl triphenyl phosphonium toxicity in drug-resistant tumor cells
}

\author{
Shrey Shah, Miller Ouellette, and Gerard G. M. D’Souza* \\ MCPHS University, School of Pharmacy-Boston, 179 Longwood Avenue, Boston, MA 02115, USA \\ Received 25 January 2022, Accepted 1 February 2022
}

\begin{abstract}
Introduction: The triphenyl phosphonium residue is a well-documented mitochondriotropic that has been shown to improve the accumulation of biomolecules in mitochondria. Stearyl triphenyl phosphonium (STPP) modified liposomes have been shown to facilitate the selective accumulation of various biomolecules in mitochondria resulting in improved effect in-vitro and in-vivo. More recently, STPP was reported to have higher toxicity towards a drug resistant ovarian cancer cell line compare to a non-drug resistant cell line. The purpose of this study was to further investigate STPP toxicity using multiple drug resistant and non-drug resistant cell lines. Methods: STPP was incorporated into phosphatidylcholine cholesterol liposomes using the thin film hydration method. Mean particle size and zeta potential was measured using dynamic light scattering. The 5,5,6,6'-tetrachloro-1,1' 3,3 ' tetraethylbenzimi-dazoylcarbocyanine iodide (JC-1) dye accumulation assay was used as an indicator of mitochondrial membrane potential in the tested cell lines. Cytotoxicity of the preparations towards different cell lines was determined using light microscopy and the CellTiter $96^{\circledR}$ AQueous One Solution Cell Proliferation assay. Results: The JC-1 accumulation assay confirmed that the drug-resistant cell lines had significantly higher dye accumulation than the non-drug resistant cell lines. Higher cytotoxicity of STPP towards drug resistant cell line was seen when incorporated into liposomes but not when dissolved in dimethyl sulfoxide (DMSO). STPP showed a comparable toxicity profile to the known oxidative phosphorylation uncoupler carbonyl cyanide p-trifluoro-methoxyphenyl hydrazone (FCCP). Discussion: Taken together, the data suggest that higher STPP toxicity in the drug-resistant cell lines is influenced by the presence of liposomal lipids and that STPP acts in a way similar to an oxidative phosphorylation uncoupler and is therefore more toxic to the drug-resistant cells that rely on a higher mitochondrial membrane potential to maintain their viability.
\end{abstract}

Keywords: Liposomes, Mitochondria, OXPHOS, Drug delivery

\section{Introduction}

Recent advances in cancer research have resulted in the addition of molecular targeted and immune-therapy approaches to the traditional chemotherapeutic approaches to cancer therapy $[1,2]$. Despite these recent advances, effective cancer therapy remains challenging. Drug resistance in particular remains a major challenge applicable to all types of therapeutic strategies $[3,4]$. In a previous study, we explored the use of mitochondriotropic liposomes to deliver paclitaxel in order to test the hypothesis that paclitaxel resistance could be bypassed in drug resistant tumor cells by increasing the accumulation of paclitaxel in mitochondria [5]. The mitochondriotropic liposomes were prepared with stearyl triphenyl phosphonium (STPP). The triphenyl phosphonium ligand is well established as a mitochondria specific targeting ligand and its conjugation to a stearyl chain allows incorporation into liposomes in a

*Corresponding author: gerard.dsouza@mcphs . edu manner to render the liposomes mitochondriotropic [6-10]. While STPP liposomes with incorporated paclitaxel did show improved cytotoxicity towards a human drug resistant ovarian cancer cell line (Ovcar-3), the data suggested that the improved cytotoxic effect in the Ovcar-3 cells was not due to increased accumulation of paclitaxel in the mitochondria but due to an apparent specific toxicity of STPP towards that cell line [5]. STPP was found to reduce mitochondrial membrane potential and change the morphological appearance of the resistant cells with no significant increase in caspase activation [5]. At the time of the study, it seemed unclear how the reduction in mitochondrial membrane potential alone could account for the specific toxicity of STPP towards the resistant cells. More recently however it has become increasingly evident that while drug sensitive tumor cells rely on the glycolytic pathway, drug-resistant tumor cells rely on increased oxidative phosphorylation (OXPHOS) to fulfill their metabolic needs $[4,11-14]$. One of the hallmarks of the increased OXPHOS activity in drug-resistant tumor cells is an increase in 
Table 1. Physical characterization of liposomal preparations.

\begin{tabular}{|c|c|c|c|c|c|}
\hline Preparation & $\begin{array}{c}\text { Lipid } \\
\text { composition }\end{array}$ & Molar ratio & $\begin{array}{l}\text { Mean particle } \\
\text { size }(\mathrm{nm})\end{array}$ & $\begin{array}{l}\text { Zeta potential } \\
(\mathrm{mV})\end{array}$ & $\begin{array}{c}\text { Polydispersity } \\
\text { index (PDI) }\end{array}$ \\
\hline Blank liposomes & PC:Chol & $70: 30$ & $96 \pm 5$ & $-7 \pm 1$ & $0.26 \pm 0.018$ \\
\hline STPP liposomes & PC:Chol:STPP & $68: 30: 2$ & $107 \pm 4$ & $28 \pm 3$ & $0.28 \pm 0.002$ \\
\hline
\end{tabular}

mitochondrial membrane potential [15]. The difference in mitochondrial membrane potential between drug-resistant and non-drug resistant tumor cells could be an explanation for how STPP exerts specific toxicity towards the resistant cell line. Based on this new finding of the metabolic differences in resistant and non-drug resistant tumor cells, we undertook the current study to further investigate the effect of STPP on mitochondrial membrane potential in drug resistant versus non-drug resistant tumor cell lines.

\section{Materials}

Human ovarian carcinoma cell lines, A2780 (non-drug resistant) and Ovcar-3 (drug resistant) and human lung carcinoma cell lines, A549 (non-drug resistant) and H69/AR (drug resistant) were purchased from American Type Cell Culture Collection (ATCC; Manassas, VA, USA). Chicken egg L- $\boldsymbol{\alpha}$-phosphatidylcholine (PC), Cholesterol (Chol) and 1,2-distearoyl-sn-glycero-3-phosphoethanolamine-N-[methoxy(polyethylene glycol)-2000(18:0 PEG 2000 PE) were purchased from Avanti Polar Lipids (Alabaster, AL, USA). Dimethyl sulfoxide (DMSO) was purchased from Sigma-Aldrich (Natick, MA, USA). Stearyl triphenyl phosphonium (STPP) was obtained from previously synthesized laboratory stock. CellTiter $96^{\circledR}$ AQueous One Solution Cell Proliferation Assay reagent was obtained from Promega (Madison, WI, USA). Carbonyl cyanide p-trifluoromethoxyphenyl hydrazone (FCCP) in DMSO and 5,5,6,6'tetrachloro-1, $1^{\prime}, 3,3^{\prime}$ tetraethylbenzimi-dazoylcarbocyanine iodide (JC-1) mitochondrial membrane potential assay kit was purchased from Abcam (Cambridge, MA, USA).

\section{Methods}

\section{Cell culture}

A2780 and A549 were cultured using RPMI 1640 medium supplemented with $1 \%$ penicillin-streptomycin (10 $000 \mathrm{U} / \mathrm{mL}$ ) and 10\% Fetal Bovine Serum (FBS). Ovcar-3 and H69/AR were cultured using Roswell Park Memorial Institute (RPMI) 1640 medium supplemented with $1 \%$ penicillin-streptomycin $(10000 \mathrm{U} / \mathrm{mL})$ and $20 \%$ FBS. Cells were cultured according to the protocol provided by ATCC in T-25 flasks that were maintained at $37^{\circ} \mathrm{C}, 5 \%$ $\mathrm{CO}_{2}$ environment.

\section{Preparation and characterization of STPP liposomes}

Liposomes having a final lipid concentration of $5 \mathrm{mg} / \mathrm{mL}$ were prepared using a thin-film hydration method. Briefly, appropriate volumes of chloroform stock solutions of egg PC, cholesterol, and/or STPP as indicated in Table 1 were added to a $10 \mathrm{~mL}$ round bottom flask. A thin lipid film was obtained after the evaporation of chloroform with the help of a rotary evaporator under vacuum at $37{ }^{\circ} \mathrm{C}$. The lipid film was then hydrated with $2 \mathrm{~mL}$ phosphate buffered saline (PBS) pH 7.4 and was subjected to sonication for two 10-min periods with a resting interval of 1 min between the two sonication periods. Sonication was carried out using a probe sonicator (Model 100 Sonic Dismembrator, VWR) with the round bottom flask immersed in an ice water bath. The size distribution and zeta potential of the prepared liposomes was determined using a 90Plus Particle Size Analyzer (Brookhaven Instruments Corporation, Holtsville, NY).

\section{Determination of mitochondrial membrane potential}

The JC-1 dye accumulation assay was used as an indicator of mitochondrial membrane potential in the tested cell lines [16-18]. Cell lines were seeded at appropriate densities (A2780 and Ovcar-3 15000 and 20000 cells/well A549 and H69/AR 16000 and 25000 cells/well) in 96 well plates and incubated overnight to yield test plates with cells at $\sim 80 \%$ confluence in each well. Media was removed from the wells and the cells were incubated with $10 \mu \mathrm{M}$ JC-1 dye for $10 \mathrm{~min}$. Following the incubation, the cells were washed once with phosphate buffered saline, $\mathrm{pH}$ 7.4(PBS), and then selected representative wells were imaged in fresh PBS using a Model BZ-X800 Analyzer (Keyence, IL, USA) all in one fluorescence microscope with appropriate filters for red and green fluorescence. Red and green fluorescence was also quantitated in wells not used for microscopy imaging with a SynergyHT microplate reader (BioTek, VT, USA).

\section{Determination of cytotoxicity}

Cytotoxicity of various preparations was determined using the CellTiter $96^{\circledR}$ AQueous One Solution Cell Proliferation Assay. Cell lines were seeded at appropriate densities (A2780 and Ovcar-3 15000 and 20000 cells/well A549 and H69/AR 16000 and 25000 cells/well) in 96 well plates and incubated overnight to yield test plates with cells at $\sim 80 \%$ confluence in each well. Preparations were added at different concentrations in $100 \mu \mathrm{L}$ of media per well and the plates were incubated for the desired exposure times. Following the incubation, preparations along with the media were removed and replaced with $100 \mu \mathrm{L}$ of media containing CellTiter $96^{\circledR}$ AQueous One Solution Cell Proliferation Assay reagent equivalent to $10 \mu \mathrm{L}$ and incubated for $1 \mathrm{~h}$ after which, the absorbance in each well was 
A
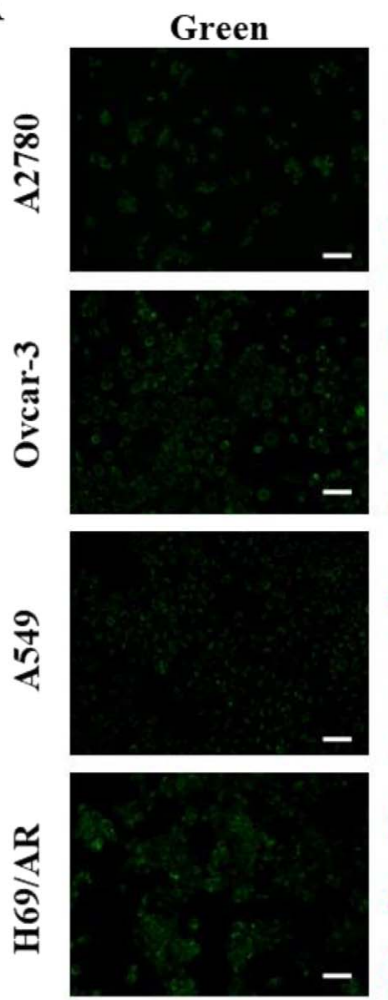

Red
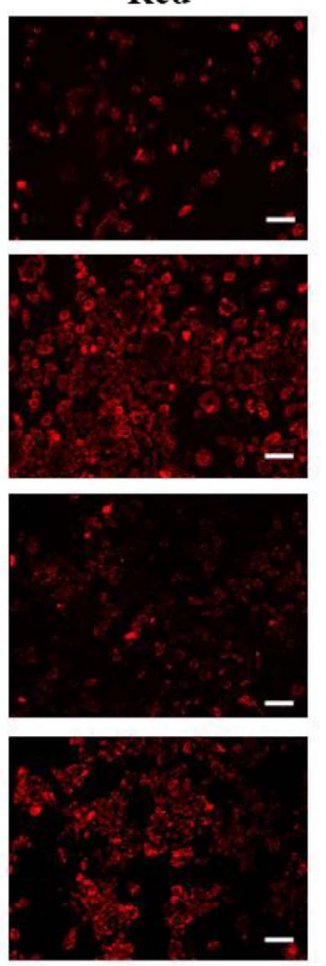

B

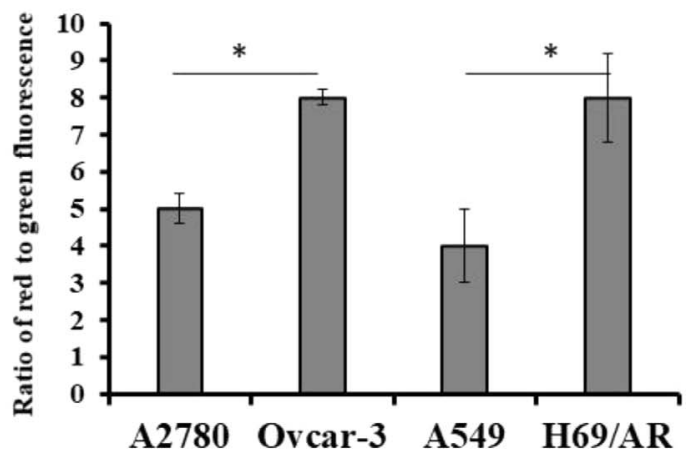

Figure 1. Determination of JC-1 Dye accumulation. A: Fluorescence micrographs with green and red filters of indicated cell lines after dye staining protocol. Scale bar represents $100 \mu \mathrm{m}$. B: Ratio of red to green fluorescence in indicated cell lines after dye staining protocol. Values represent mean \pm standard deviation from 3 independent experiments. ${ }^{*} p<0.05$, Student's $t$ test.

measured at $490 \mathrm{~nm}$ in a SynergyHT microplate reader (BioTek, VT, USA).

Representative light microscopy images of cells post treatment were captured from selected wells using a Model BZ-X800 Analyzer (Keyence, IL, USA).

\section{Results}

\section{Physical characteristics of STPP formulations}

All liposomal preparations used in the study were characterized for their size, zeta potential and PDI. The values for each parameter in Table 1 are represented as average \pm standard deviation from three independent preparations. All measured values are within the expected range for preparations of this type.

\section{Mitochondrial membrane potential status of cell lines}

Figure 1 shows representative fluorescence micrographs of JC-1 dye staining in A2780, Ovcar-3, A549, and H69/AR cell line. JC-1 is a green fluorescent dye that dimerizes at high concentration. JC-1 is well known to accumulate in mitochondria of cells that have a high mitochondrial membrane potential and reach a high enough concentration in the mitochondrial network to result in the dimerization of the dye molecules [16-18]. In cells with mitochondria that have low membrane potential JC-1 does not accumulate at a high enough concentration to dimerize. When dimerized, JC-1 exhibits red fluorescence. It is therefore possible to compare the mitochondrial membrane potential of cells based on the red:green fluorescence ratio after staining with JC-1. As seen from Figure 1A, Ovcar-3 and H69/AR cell lines have higher red fluorescence in comparison with A2780 and A549 respectively. Figure 1B shows the quantitative estimation of JC-1 dye fluorescence in A2780, Ovcar3, A549, and H69/AR cell lines. The red:green fluorescence ratio of Ovcar-3 and H69/AR was significantly higher compared with A2780 and A549 respectively $(p<0.05$ Student's $t$ test).

\section{Cytotoxic effect of STPP preparations}

The toxicity of STPP DMSO solution, STPP liposomes and STPP DMSO solution co-administered with plain liposomes towards the four cell lines was measured using a metabolic assay. The goal of these experiments was to allow not only for the comparison of cytotoxicity in drug-resistant versus non-drug resistant cell lines but also to investigate any potential influence of exposure time or delivery vehicle on the effect of STPP in the selected cell lines. Figure 2 shows that STPP liposomes and co-delivery of blank liposomes with STPP in DMSO exhibit a greater toxic effect towards the drug resistant Ovcar-3 cell line compared to the non-drug resistant A2780 cell line. A significantly higher $(p<0.05$, Student's $t$ test $)$ toxicity towards Ovcar-3 cells 
A

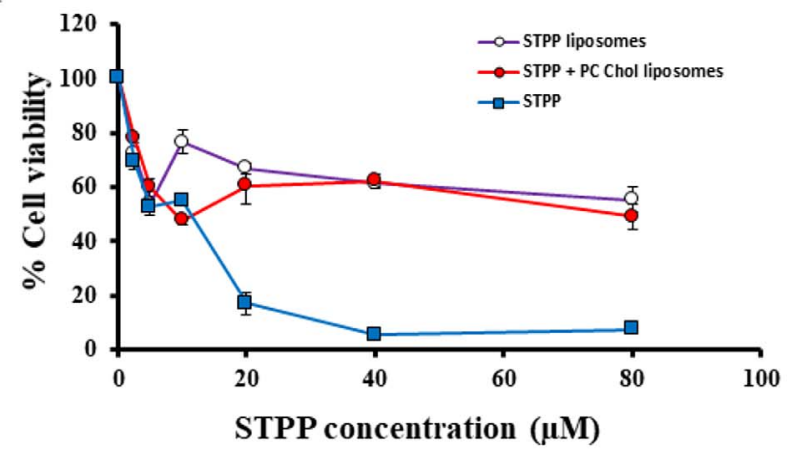

C

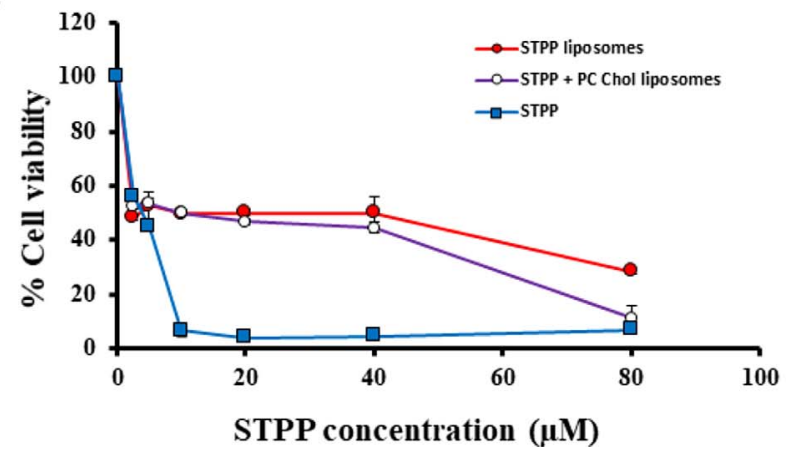

B

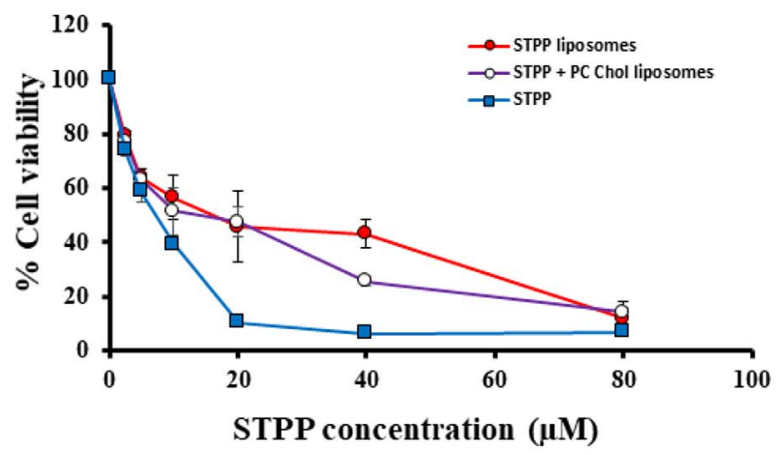

D

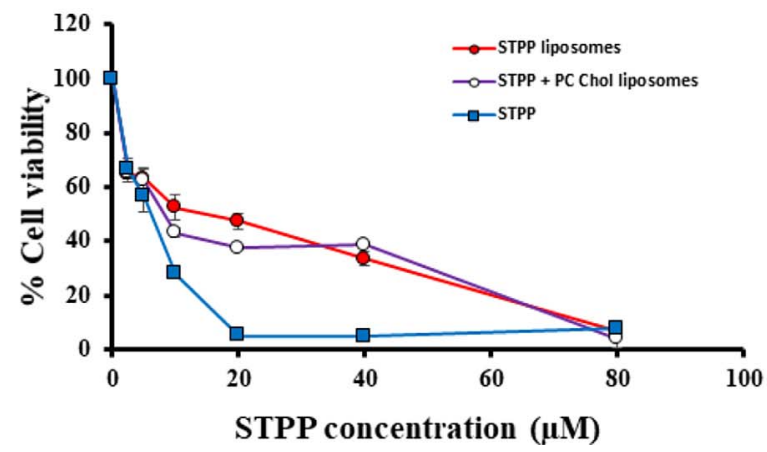

Figure 2. Quantitative determination of STPP toxicity in human ovarian carcinoma cells. Percent cell viability in A, C: A2780 cells and B, D: Ovcar-3 cells, treated with STPP liposomes, STPP in DMSO and STPP in DMSO mixed with PC:Cholesterol liposomes for A, B: $5 \mathrm{~h}$ and C, D: $12 \mathrm{~h}$ at indicated concentrations of STPP. Values represent mean \pm standard deviation from three independent experiments.

occurs at a wider range of concentrations ( $5 \mu \mathrm{M}$ and above) at $5 \mathrm{~h}$ in comparison with $12 \mathrm{~h}(40 \mu \mathrm{M}$ and above). Likewise Figure 3 shows that STPP liposomes and co-delivery of blank liposomes with STPP in DMSO exhibit a greater toxic effect towards the drug resistant cell line H69/AR compared to the non-drug resistant A549 cell line. A significantly higher $(p<0.05$, Student's $t$ test) toxicity towards $\mathrm{H} 69 / \mathrm{AR}$ occurs at all the tested concentrations at $5 \mathrm{~h}$, however, at $12 \mathrm{~h}$ it only occurs from $10 \mu \mathrm{M}$ to $40 \mu \mathrm{M}$. STPP in DMSO did not show a greater toxic effect towards any of the drug resistant cell lines compared to the corresponding non-drug resistant cell lines. For additional mechanistic evaluation of the STPP effect in the selected cell lines, the known uncoupler FCCP was also tested in an analogous manner to that of STPP. As can be seen from Figure 4, FCCP shows a significantly higher $(p<0.05$, Student's $t$ test) level of toxicity towards the drug resistant H69/AR cell line compared to the non-drug resistant A549 cell line. No significant difference was observed in the toxicity of FCCP towards Ovcar-3 and A2780 cell lines. The light micrographs in Figure 5 show that the morphological changes in the treated cells resulting from STPP treatments are similar to those resulting from FCCP treatment. The morphology of the drug resistant cell lines is also clearly different than that of the non-drug resistant cell lines with both STPP and FCCP treatments.

\section{Discussion}

The development of intrinsic or acquired drug resistance by tumor cells continues to limit the effectiveness of clinical therapy for many tumor types [19, 20]. Although, several mechanisms for the development of drug resistance in the tumor have been identified, the general approaches for over-coming tumor drug-resistance focus on either inhibiting molecular events underlying resistance or using particulate carrier systems for increasing the drug accumulation at the target site [21-27]. Recent advances in understanding the cellular metabolism of drug resistant tumor cells, suggest that contrary to the prevailing Warburg theory that tumor cells rely on aerobic glycolysis, certain drug-resistant tumor cells in fact rely on increased OXPHOS activity for their survival $[4,11,12,28-30]$. These recent findings offered a new insight to previous studies [5] in which STPP appeared to have a greater level of toxicity toward drug resistant ovarian carcinoma Ovcar-3 tumor cells compared to non-drug resistant ovarian carcinoma A2780 cells. At that time, experiments aimed at elucidating the mechanism of cell death triggered by STPP confirmed a reduction in the mitochondrial membrane potential as well change in the morphological appearance; however, the morphological changes and Caspase activity profile were found to be distinct from that of camptothecin suggesting a non-apoptotic 
A

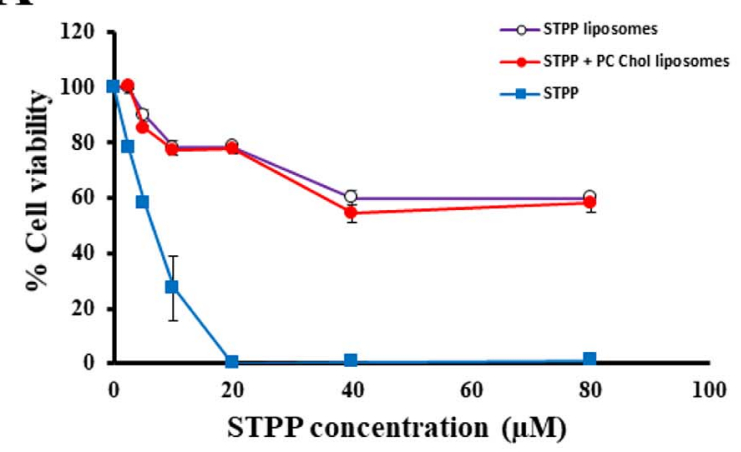

C

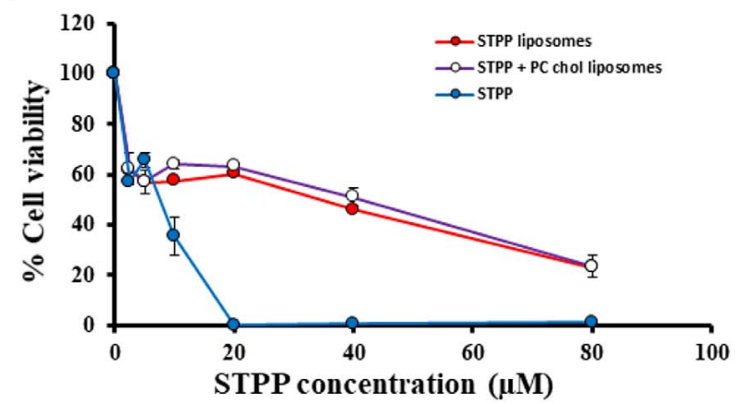

B

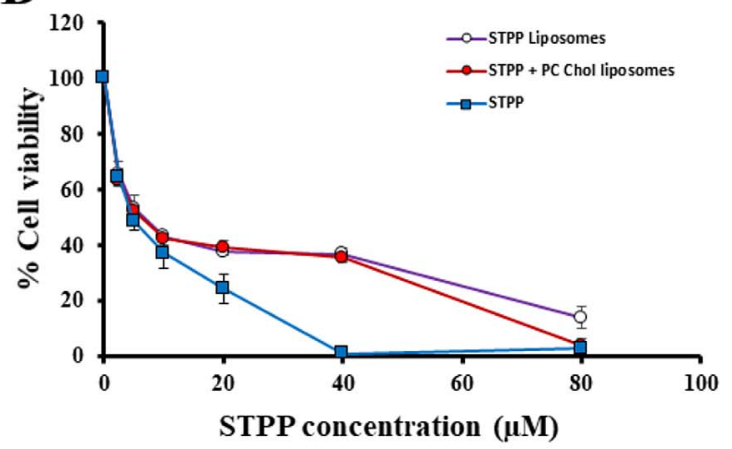

D

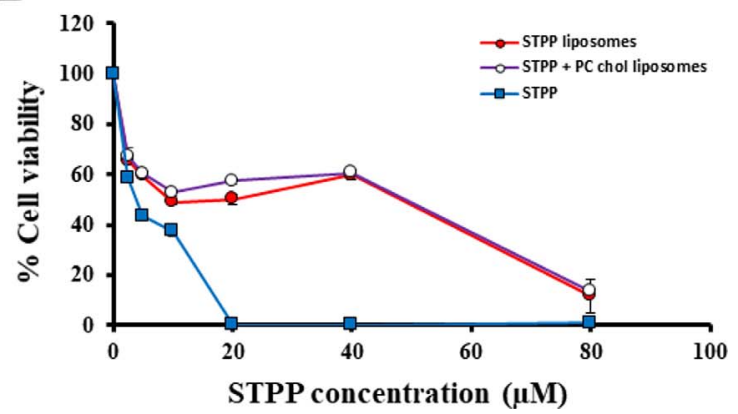

Figure 3. Quantitative determination of STPP toxicity in human lung carcinoma cells. Percent cell viability in A, C: A549 cells and B, D: H69/AR cells, treated with STPP liposomes, STPP in DMSO and STPP in DMSO mixed with PC: Cholesterol liposomes for A, B: $5 \mathrm{~h}$ and C, D: $12 \mathrm{~h}$ at indicated concentrations of STPP. Values represent mean \pm standard deviation from three independent experiments.
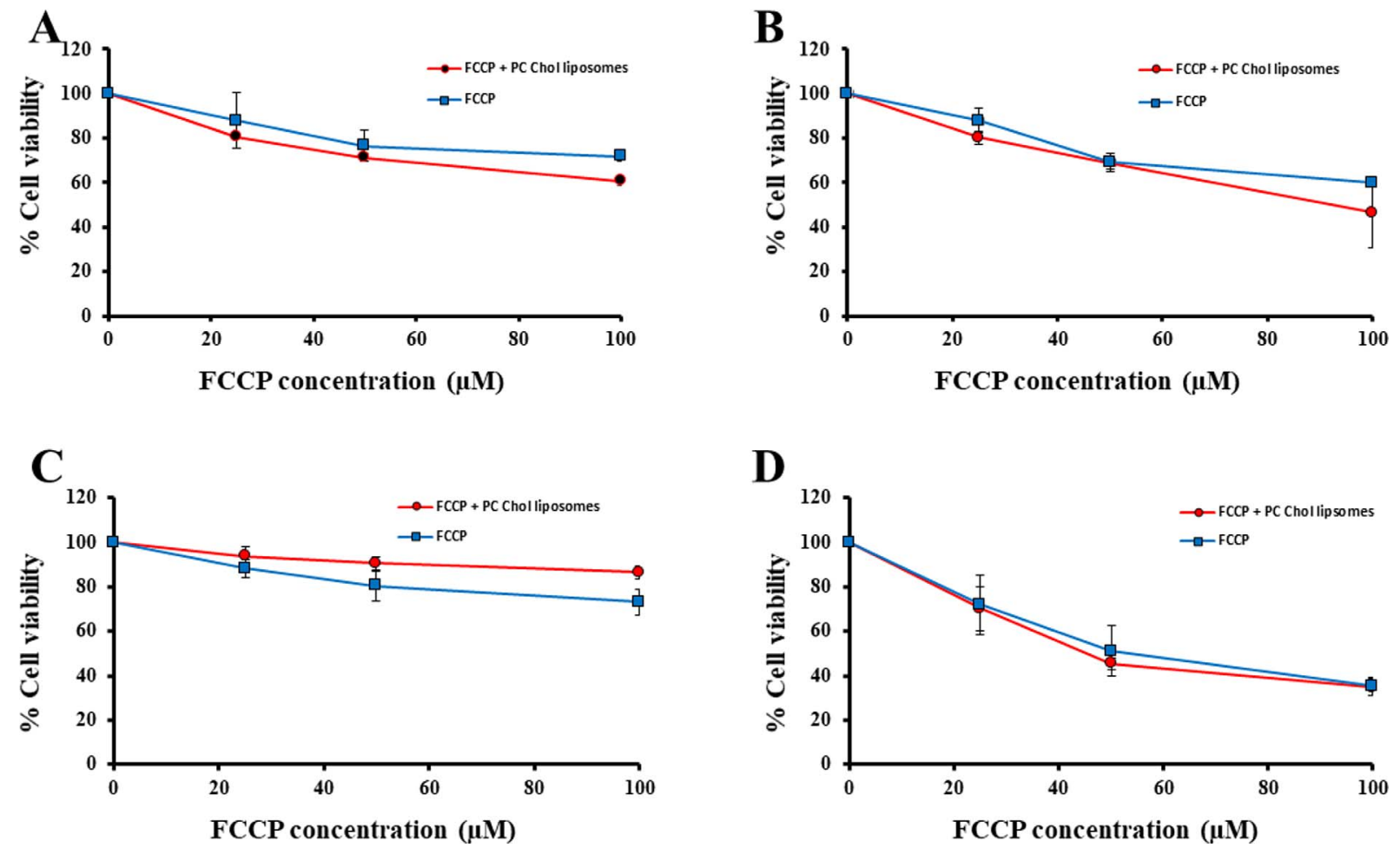

Figure 4. Quantitative determination of FCCP toxicity in human ovarian carcinoma and human lung carcinoma cells. Percent cell viability in A: A2780 cells, B: Ovcar3 cells, C: A549 cells, D: H69/AR cells exposed to either FCCP in DMSO or FCCP in DMSO with PC: Cholesterol liposomes for $4 \mathrm{~h}$ at indicated concentrations of FCCP. Values represent mean \pm standard deviation from three independent experiments. 


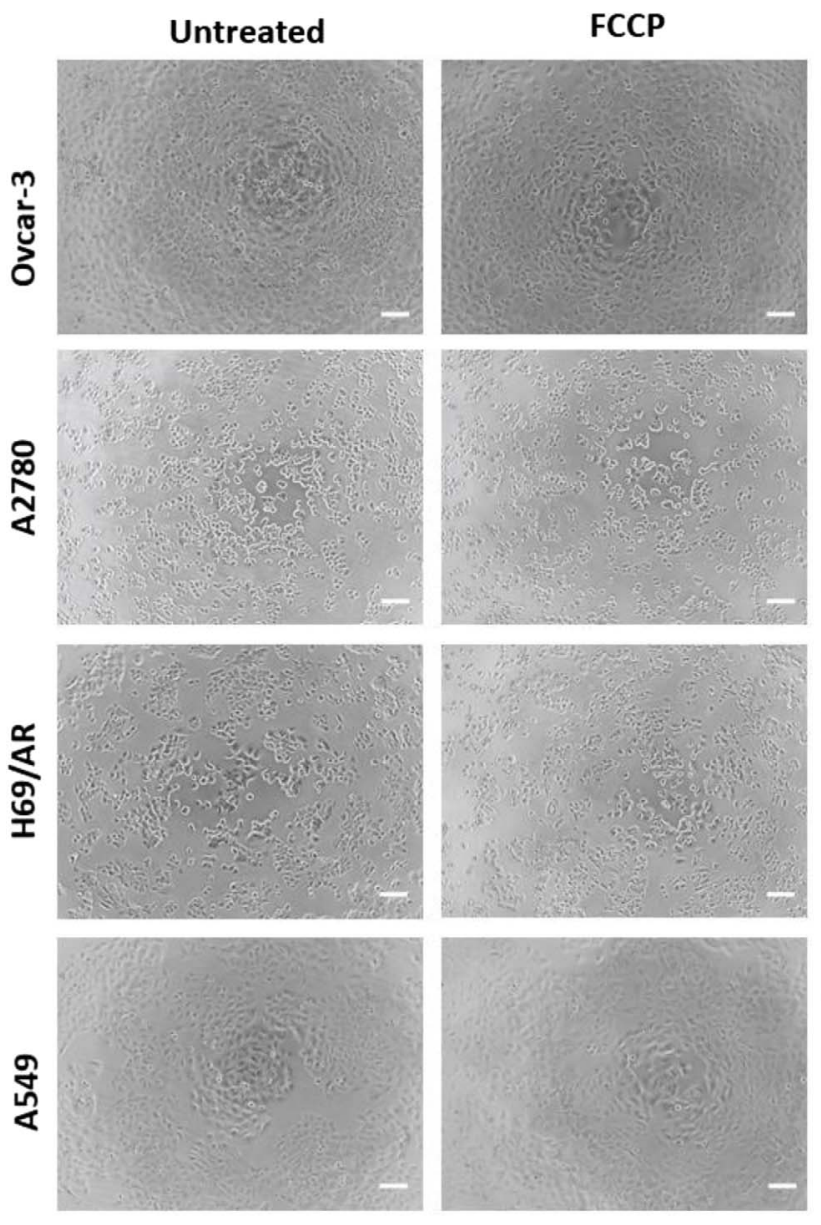

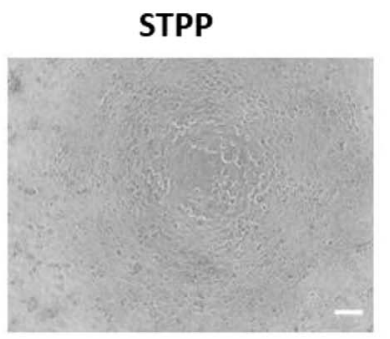

\section{STPP liposomes}
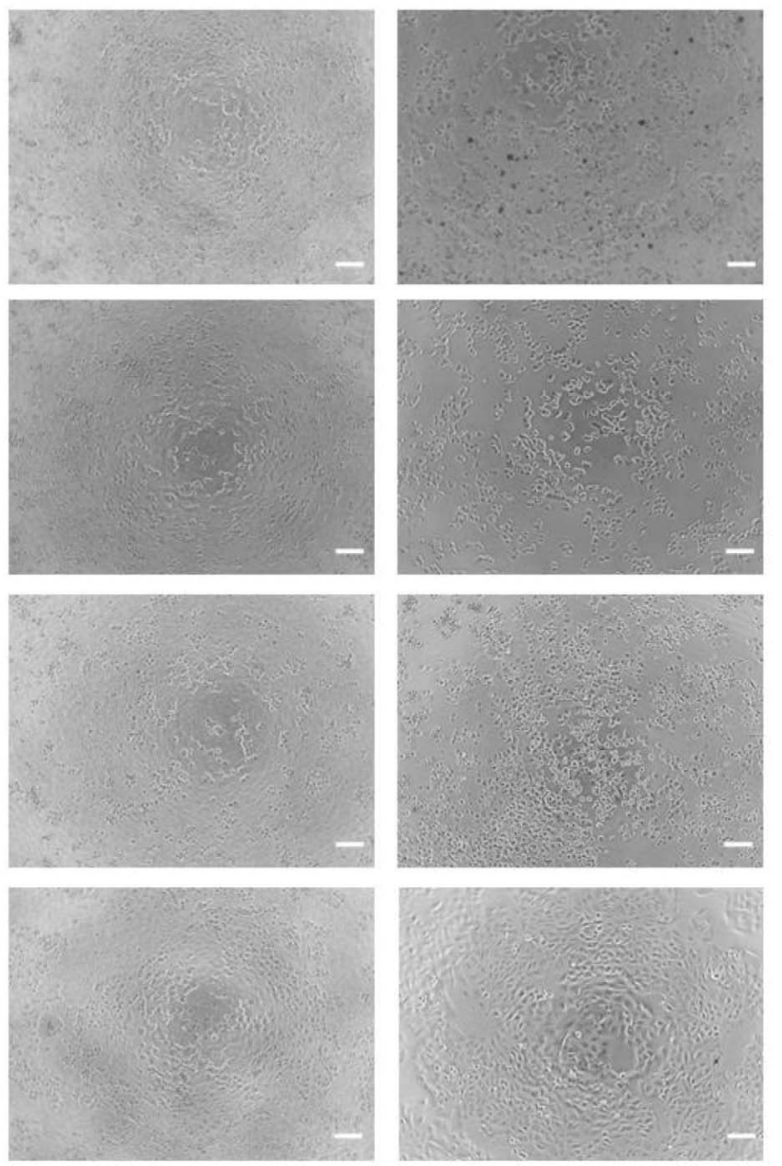

Figure 5. Qualitative determination of STPP and FCCP toxicity human ovarian carcinoma and human lung carcinoma cells. Light micrographs of indicated cell lines with no treatment (untreated) $100 \mu \mathrm{M}$ FCCP in DMSO for $4 \mathrm{~h}(\mathrm{FCCP}), 80 \mu \mathrm{M}$ STPP in DMSO for $5 \mathrm{~h}$ (STPP) and $80 \mu \mathrm{M}$ STPP liposomes for $5 \mathrm{~h}$ (STPP liposomes). Scale bar indicates $100 \mu \mathrm{m}$.

mechanism of cell death [5]. The aim of this study was to test the hypothesis that STPP acts like an OXPHOS uncoupler and, therefore, has a more pronounced cytotoxic effect in the drug-resistant cell lines that are now known to rely on higher levels of OXPHOS for cellular activity.

An additional pair of drug-resistant (H69/AR) and nondrug resistant (A549) cell lines was included to check that the differential toxicity was not limited to the original pair of drug-resistant (Ovcar-3) and non-drug resistant (A2780) cells. The JC-1 assay confirmed that both drug-resistant cell lines had higher levels of JC-1 accumulation compared to their non-drug resistant counterparts, strongly suggesting the existence of a higher mitochondrial membrane potential in the drug resistant cell lines. STPP liposomes showed higher toxicity towards both the drug resistant cell lines. STPP in DMSO was used as a non-liposomal delivery control and unexpectedly not only was the toxicity of STPP much higher when administered in DMSO, no differential toxicity was observed in either of the cell pairs tested. This finding raised the possibility that the liposomal lipids might have some role in the effect of STPP and prompted the testing of a mixture of plain (PC:Chol) liposomes with STPP dissolved in DMSO. This additional control group showed no significantly different cytotoxic profile compared to STPP incorporated in liposomes. Taken together, these observations seem to suggest a potential role for liposomal lipids in the toxicity of STPP.

When the cytotoxic effect of the well-known OXPHOS uncoupler FCCP was evaluated, the results showed a similar differential toxicity towards the drug resistant lung carcinoma cell line but not in the drug resistant ovarian carcinoma cell line. The presence of liposomal lipids did not result in any significant changes in the cytotoxic profile of FCCP towards the cell lines tested. Morphological changes resulting from either STPP or FCCP exposure appear similar. It would therefore seem that due to its previously reported effect on lowering mitochondrial membrane potential [8], STPP essentially acts like an OXPHOS uncoupler and the presence of liposomal lipids is essential to its selective toxicity towards the drug resistant cell lines.

Structurally, with the triphenyl phosphonium group serving as a head group and the stearyl chain as a hydrophobic tail, STPP is very similar to a lysophospholipid. Lysophospholipids are well documented to exert a permeability increasing effect on lipid bilayers [31, 32]. It is therefore likely that the accumulation of STPP in the 
mitochondrial membrane has a permeability altering effect that leads to the loss of mitochondrial membrane potential and effective OXPHOS uncoupling. FCCP in contrast, is known to exert its OXPHOS uncoupling effect by serving as a proton transporter across the mitochondrial membranes [33]. The independence of FCCP effect from the presence of liposomal phospholipids also suggests a different mechanism for STPP uncoupling. While it cannot entirely be ruled out that the presence of liposomal lipids could alter the bioenergetic state of the drug resistant cells on account of lipid metabolism, a lysophospholipid-like permeability altering mechanism for STPP toxicity is more likely to depend on the influence of liposomal lipids on its membrane interactions and trafficking within the cell. Lysophospholipids have been shown to have different effects on membranes depending on whether they are symmetrically distributed in the bilayer or are concentrated in the external layer [34-36]. Given the well documented role of naturally occurring lysophospholipids in membrane permeabilizing and membrane fusion effects [36-38], it is certainly reasonable to hypothesize similar effects for STPP. When incorporated in liposomes, it is very likely that STPP has sufficient opportunity to distribute between both layers of the liposomal bilayer. The subsequent interactions of the liposomal membrane and the cellular membrane ensure that STPP gets trafficked into the cell in endosomal membranes in which it remains symmetrically distributed eventually reaching the mitochondrial membrane where it likely maintains its symmetrical distribution in the inner and outer leaflets of the bilayer. If administered in a DMSO solution, it is more likely that all STPP molecules localize to the external leaflet of the cell membrane and thereby exert a different effect at the cell membrane and when eventually transported to the mitochondrial membrane. Further support for this hypothetical mechanism can be drawn from a previous study comparing the toxicity of STPP with triphenyl phosphonium (TPP) modified phospholipids [8]. TPP modified phospholipids showed comparable mitochondrial accumulation to STPP without the mitochondrial membrane potential reducing effect and its associated cytotoxicity [8]. These findings are therefore a good cautionary tale about choosing an appropriate lipid anchor to enable surface modification of liposomes with potential targeting ligands. Finally, since the selective toxicity of STPP towards drug resistant cells occurs at concentrations that would be difficult to achieve in vivo in solid tumors [39, 40], further investigation of STPP as a potential tumor therapy agent is not warranted.

\section{Nomenclature}

$\begin{array}{ll}\text { Chol } & \text { Cholesterol } \\ \text { DMSO } & \text { Dimethyl sulfoxide } \\ \text { FBS } & \text { Fetal bovine serum } \\ \text { FCCP } & \text { Carbonyl cyanide p-trifluoro-methoxyphenyl } \\ & \text { hydrazone } \\ \text { JC-1 } & 5,5,6,6^{\prime} \text {-tetrachloro- } 1,1^{\prime}, 3,3^{\prime} \text { tetraethylbenzimi- } \\ & \text { dazoylcarbocyanine iodide }\end{array}$

OXPHOS Oxidative phosphorylation

PBS Phosphate buffered saline

PC L- $\alpha$-phosphatidylcholine

PC-Chol L- $\boldsymbol{\alpha}$-phosphatidylcholine-cholesterol

PDI Polydispersity index

RPMI Roswell Park Memorial Institute (RPMI) 1640 Medium

STPP Stearyl triphenyl phosphonium

\section{Conflict of interest}

The authors have no conflict of interest to declare.

\section{Acknowledgments}

This work was entirely supported by funding from the MCPHS University, School of Pharmacy-Boston.

\section{References}

1. Vanneman M, Dranoff G (2012), Combining immunotherapy and targeted therapies in cancer treatment. Nat Rev Cancer 12(4), 237-251.

2. Zhang Y, Zhang Z (2020), The history and advances in cancer immunotherapy: understanding the characteristics of tumor-infiltrating immune cells and their therapeutic implications. Cell Mol Immunol 17(8), 807-821.

3. Housman G, Byler S, Heerboth S, Lapinska K, Longacre M, Snyder N, Sarkar S (2014), Drug resistance in cancer: an overview. Cancers (Basel) 6(3), 1769-1792.

4. Bosc C, Selak MA, Sarry JE (2017), Resistance is futile: targeting mitochondrial energetics and metabolism to overcome drug resistance in cancer treatment. Cell Metab 26(5), 705-707.

5. Solomon MA, Shah AA, D'Souza GG (2013), In vitro assessment of the utility of stearyl triphenyl phosphonium modified liposomes in overcoming the resistance of ovarian carcinoma Ovcar-3 cells to paclitaxel. Mitochondrion 13(5), 464-472.

6. Boddapati SV, Tongcharoensirikul P, Hanson RN, D'Souza GG, Torchilin VP, Weissig V (2005), Mitochondriotropic liposomes. J Liposome Res 15(1-2), 49-58.

7. Patel NR, Hatziantoniou S, Georgopoulos A, Demetzos C, Torchilin VP, Weissig V, D'Souza GG (2010), Mitochondriatargeted liposomes improve the apoptotic and cytotoxic action of sclareol. J Liposome Res 20(3), 244-249.

8. Benien P, Solomon MA, Nguyen P, Sheehan EM, Mehanna AS, D'Souza GG (2016), Hydrophobized triphenyl phosphonium derivatives for the preparation of mitochondriotropic liposomes: choice of hydrophobic anchor influences cytotoxicity but not mitochondriotropic effect. J Liposome Res 26(1), 21-27.

9. Biswas S, Dodwadkar NS, Deshpande PP, Torchilin VP (2012), Liposomes loaded with paclitaxel and modified with novel triphenylphosphonium-PEG-PE conjugate possess low toxicity, target mitochondria and demonstrate enhanced antitumor effects in vitro and in vivo. J Control Release 159(3), 393-402.

10. Benien P, Almuteri M, Shah S, Böhlke M, Mehanna A, D'Souza GG (2021), Synthesis of triphenylphosphonium phospholipid conjugates for the preparation of mitochondriotropic liposomes, in: V Weissig, M Edeas (Eds.), Mitochondrial Medicine : Volume 1: Targeting Mitochondria, Springer US, New York, NY, pp. 119-126. 
11. De Francesco EM, Ózsvári B, Sotgia F, Lisanti MP (2019), Dodecyl-TPP targets mitochondria and potently eradicates cancer stem cells (CSCs): synergy with FDA-approved drugs and natural compounds (vitamin $\mathrm{C}$ and berberine). Front Oncol 9, 615 .

12. Viale A, Pettazzoni P, Lyssiotis CA, Ying H, Sánchez N, Marchesini M, Carugo A, Green T, Seth S, Giuliani V, KostAlimova M (2014), Oncogene ablation-resistant pancreatic cancer cells depend on mitochondrial function. Nature 514 (7524), 628-632.

13. Matassa DS, Amoroso MR, Lu H, Avolio R, Arzeni D, Procaccini C, Faicchia D, Maddalena F, Simeon V, Agliarulo I, Zanini E (2016), Oxidative metabolism drives inflammation-induced platinum resistance in human ovarian cancer. Cell Death Differ 23(9), 1542-1554.

14. Lee KM, Giltnane JM, Balko JM, Schwarz LJ, GuerreroZotano AL, Hutchinson KE, Nixon MJ, Estrada MV, Sánchez V, Sanders ME, Lee T (2017), MYC and MCL1 cooperatively promote chemotherapy-resistant breast cancer stem cells via regulation of mitochondrial oxidative phosphorylation. Cell Metab 26(4), 633-647.e7.

15. Chang SY, Lee MY, Chung PS, Kim S, Choi B, Suh MW, Rhee CK, Jung JY (2019), Enhanced mitochondrial membrane potential and ATP synthesis by photobiomodulation increases viability of the auditory cell line after gentamicininduced intrinsic apoptosis. Sci Rep 9(1), 19248.

16. Reers M, Smiley ST, Mottola-Hartshorn C, Chen A, Lin M, Chen LB (1995), Mitochondrial membrane potential monitored by JC-1 dye. Methods Enzymol 260, 406-417.

17. Cossarizza A, Baccaranicontri M, Kalashnikova G, Franceschi C (1993), A new method for the cytofluorimetric analysis of mitochondrial membrane potential using the J-aggregate

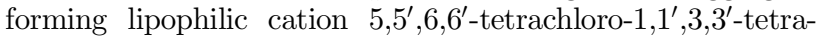
ethylbenzimidazolcarbocyanine iodide (JC-1). Biochem Biophys Res Commun 197(1), 40-45.

18. Sivandzade F, Bhalerao A, Cucullo L (2019), Analysis of the mitochondrial membrane potential using the cationic JC-1 dye as a sensitive fluorescent probe. Bio Protoc 9(1), PMID: 30687773 .

19. Gottesman MM, Lavi O, Hall MD, Gillet JP (2016), Toward a better understanding of the complexity of cancer drug resistance. Annu Rev Pharmacol Toxicol 56, 85-102.

20. Brozovic A, Gonçalves AC, Jurkovicova D, Linē A, Machuqueiro M, Saponara S, Sarmento-Ribeiro AB, Xavier CP, Vasconcelos MH (2019), The multi-factorial nature of clinical multidrug resistance in cancer. Drug Resist Updat 46, 100645 .

21. Nobili S, Landini I, Giglioni B, Mini E (2006), Pharmacological strategies for overcoming multidrug resistance. Curr Drug Targets 7(7), 861-879.

22. Shukla S, Wu CP, Ambudkar SV (2008), Development of inhibitors of ATP-binding cassette drug transporters: present status and challenges. Expert Opin Drug Metab Toxicol 4(2), 205-223.

23. Nanayakkara AK, Follit CA, Chen G, Williams NS, Vogel PD, Wise JG (2018), Targeted inhibitors of P-glycoprotein increase chemotherapeutic-induced mortality of multidrug resistant tumor cells. Sci Rep 8(1), 967.

24. Elshimali YI, Wu Y, Khaddour H, Wu Y, Gradinaru D, Sukhija H, Chung SS, Vadgama JV (2018), Optimization of cancer treatment through overcoming drug resistance. J Cancer Res Oncobiol 1(2), PMID: 29932172.

25. Chan MS, Liu LS, Leung HM, Lo PK (2017), Cancer-cellspecific mitochondria-targeted drug delivery by dual-ligandfunctionalized nanodiamonds circumvent drug resistance. ACS Appl Mater Interfaces 9(13), 11780-11789.

26. Dong X, Mumper RJ (2010), Nanomedicinal strategies to treat multidrug-resistant tumors: current progress. Nanomedicine (Lond) 5(4), 597-615.

27. Yuan Y, Cai T, Xia X, Zhang R, Chiba P, Cai Y (2016), Nanoparticle delivery of anticancer drugs overcomes multidrug resistance in breast cancer. Drug Deliv 23(9), 3350-3357.

28. Wolf DA (2014), Is reliance on mitochondrial respiration a "chink in the armor" of therapy-resistant cancer? Cancer Cell 26(6), 788-795.

29. Lee JS, Lee H, Jang H, Woo SM, Park JB, Lee SH, Kang JH, Kim HY, Song J, Kim SY (2020), Targeting oxidative phosphorylation reverses drug resistance in cancer cells by blocking autophagy recycling. Cells 9(9), PMID: 32883024.

30. Hirpara J, Eu JQ, Tan JKM, Wong AL, Clement MV, Kong LR, Ohi N, Tsunoda T, Qu J, Goh BC, Pervaiz S (2019), Metabolic reprogramming of oncogene-addicted cancer cells to OXPHOS as a mechanism of drug resistance. Redox Biol 25, 101076.

31. van Zutphen H, van Deenen LLM (1967), The effect of lysolecithin on the electrical resistance of lecithin bilayer membranes. Chem Phys Lipids 1(4), 389-391.

32. Lee Y, Chan SI (1977), Effect of lysolecithin on the structure and permeability of lecithin bilayer vesicles. Biochemistry 16 (7), 1303-1309.

33. Benz R, McLaughlin S (1983), The molecular mechanism of action of the proton ionophore FCCP (carbonylcyanide p-trifluoromethoxyphenylhydrazone). Biophys J 41(3), 381398.

34. Chernomordik L, Chanturiya A, Green J, Zimmerberg J (1995), The hemifusion intermediate and its conversion to complete fusion: regulation by membrane composition. Biophys J 69(3), 922-929.

35. Gunther-Ausborn S, Praetor A, Stegmann T (1995), Inhibition of influenza-induced membrane fusion by lysophosphatidylcholine. J Biol Chem 270(49), 29279-29285.

36. Wu H, Zheng L, Lentz BR (1996), A slight asymmetry in the transbilayer distribution of lysophosphatidylcholine alters the surface properties and poly(ethylene glycol)-mediated fusion of dipalmitoylphosphatidylcholine large unilamellar vesicles. Biochemistry 35(38), 12602-12611.

37. Chernomordik L, Kozlov MM, Zimmerberg J (1995), Lipids in biological membrane fusion. J Membr Biol 146(1), $1-14$.

38. Fuller N, Rand RP (2001), The influence of lysolipids on the spontaneous curvature and bending elasticity of phospholipid membranes. Biophys J 81(1), 243-254.

39. Milas L, Hunter NR, Kurdoglu B, Mason KA, Meyn RE, Stephens LC, Peters LJ (1995), Kinetics of mitotic arrest and apoptosis in murine mammary and ovarian tumors treated with taxol. Cancer Chemother Pharmacol 35(4), 297-303.

40. Jang SH, Wientjes MG, Au JL (2001), Determinants of paclitaxel uptake, accumulation and retention in solid tumors. Invest New Drugs 19(2), 113-123. 